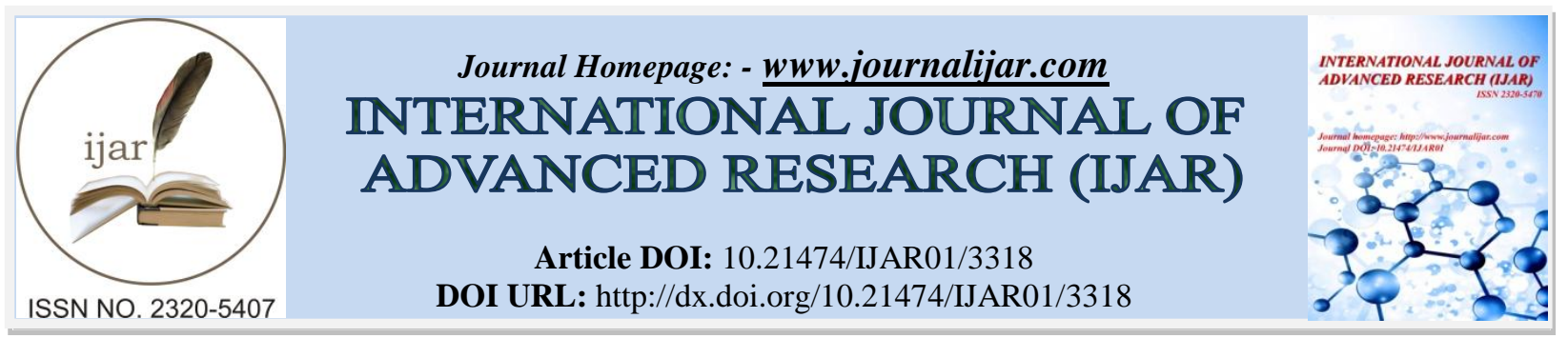

RESEARCH ARTICLE

\title{
WEYL FRACTIONAL DERIVATIVE OF THE PRODUCT MULTIVARIABLES POLYNOMIALS AND I -FUNCTION.
}

\section{Harish Kumar Mishra.}

Department of University Institute of Engineering\& Technology, Babasaheb Bhimrao Ambedkar University, Lucknow, India.

\section{Manuscript Info}

Manuscript History

Received: 20 December 2016

Final Accepted: 28 January 2017

Published: February 2017

\section{Abstract}

In this research work, we establish a theorem on Weyl fractional derivative of the product multivariable polynomials and I -function . Certain special cases of our theorem have been discussed .

Mathematics Subject classification - 26A33,33C 60, 44A15.

Key words:-

Weyl fractional derivative operator, multivariable polynomials and I-function

Copy Right, IJAR, 2017,. All rights reserved.

\section{Introduction:-}

The I- function of the one variable is defined by Saxena (1982) and we will represent here in the following manner:

$$
\begin{aligned}
& \mathrm{I}[\mathrm{z}]=\mathrm{I}_{p_{i}, q_{i} ; r}^{m, n}[z]=\mathrm{I}_{p_{i}, q_{i} ; r}^{m, n}\left[z \mid \begin{array}{l}
\ldots, \ldots \\
\ldots, \ldots
\end{array}\right]=\mathrm{I}_{p_{i}, q_{i} ; r}^{m, n}\left[z \mid \begin{array}{ll}
\left(a_{j}, e_{j}\right)_{1, n} ; & \left(a_{j i} e_{j i}\right)_{n+1, p i} \\
\left(b_{j}, f_{j}\right)_{1, m} ; & \left(b_{j i} f_{j i}\right)_{m+1, q i}
\end{array}\right] \\
& =\frac{1}{2 \pi i} \int_{L} \theta(s) z^{s} d s,
\end{aligned}
$$

where $i=\sqrt{(-1)}, z(\neq 0)$ is a complex variable and (1.2) $z^{s}=\exp [s\{\log |z|+i \arg z\}]$.In which $\log |z|$ represent the natural $\operatorname{logarithm}$ of $|z|$ and $\arg |z|$ is not necessarily the principle value. An empty product is interpreted as unity. Also,

$$
\theta(s)=\frac{\prod_{j=1}^{m} \Gamma\left(b_{j}-f_{j} s\right) \prod_{j=1}^{n} \Gamma\left(1-a_{j}+e_{j} s\right)}{\sum_{i=1}^{r}\left[\prod_{j=m+1}^{q_{i}} \Gamma\left(1-b_{j i}+f_{j i} s\right) \prod_{j=n+1}^{p_{i}} \Gamma\left(a_{j i}-e_{j i} s\right)\right]}, \mathrm{m}, \mathrm{n}, \mathrm{p}_{\mathrm{i}} \text { and } q_{i} \forall i \in(1, \ldots r) \text { are non-negative }
$$

integers satisfying $0 \leq n \leq p_{i}, 0 \leq m \leq q_{i} ; \forall i \in\{1, \ldots, r\}, e_{j i},\left(j=1, \ldots, p_{i} ; i=1, \ldots r\right)$ and $f_{j i},\left(j=1, \ldots, q_{i} ; i=1, \ldots r\right)$ are assumed to be positive quantities for standardization purpose. Also $a_{j i},\left(j=1, \ldots, p_{i} ; i=1, \ldots r\right)$ and $b_{j i},\left(j=1, \ldots, q_{i} ; i=1, \ldots, r\right)$ are complex numbers such that none of the points. 


$$
S=\left\{\left(b_{n}+v\right) \mid f_{h}\right\}, h=1, \ldots, m ; v=0,1,2, \ldots,
$$

which are the poles of $\Gamma\left(b_{h}-f_{h} s\right), h=1, \ldots, m$ and the points

$$
S=\left\{\left(a_{l}-n-1\right) \mid e_{l}\right\} l=1, \ldots, n ; \eta=0,1,2, \ldots,
$$

Which poles are of $\Gamma\left(1-a_{l}+e_{l} s\right)$ coincide with one another, i.e. with

$$
e_{l}\left(b_{n}+v\right) \neq b_{n}\left(a_{l}-\eta-1\right),
$$

for $v, \eta=0,1,2, \ldots ; h=1, \ldots, m ; l=1, \ldots, n$.

Further, the contour $\mathrm{L}$ runs from $-i_{\infty}$ to $+i_{\infty}$. Such that the poles of $\Gamma\left(b_{n}-s\right), h=1, \ldots, m$; lie to the right of $\mathrm{L}$ and the poles $\Gamma\left(1-a_{l}+e_{l} s\right), l=1, \ldots, n$ lie to the left of $\mathrm{L}$. The integral converges, if $|\operatorname{argz}|$ $<\frac{1}{2} B \pi, B>0, A \leq 0$, where

$$
\begin{aligned}
& A=\sum_{j=1}^{p_{i}} e_{j i}-\sum_{j=1}^{q_{i}} f_{j i} \text { and } \\
& B=\sum_{j=1}^{n} e_{j}-\sum_{j=n+1}^{p_{i}} e_{j i}+\sum_{j=1}^{m} f_{j}-\sum_{j=m+1}^{q_{i}} f_{j i} \quad \forall i \in(1, \ldots, r)
\end{aligned}
$$

Let A denote a class of good functions. By good function f, we mean Miller [1975, p.82] a function which is everywhere differentiable any number of times and if it is all of its derivatives are $0\left(x^{-v}\right)$, for all $v$ as $\mathrm{x}$ in increases without limit. We define the Weyl fractional derivatives of a function $\mathrm{g}(\mathrm{z})$ as follows:-

Let $g \in A$, then for $q<0$,

$$
{ }_{z} W_{\infty}^{q} g(z)=\frac{(-1)^{q}}{\Gamma(-q)} \int_{z}^{\infty}(u-z)^{-q-1} g(u) d u .
$$

For $q \geq 0$

(1.10) $\quad{ }_{z} W_{\infty}^{q} g(z)=\frac{d^{n}}{d z^{n}}\left({ }_{z} W_{\infty}^{q-n} g(z)\right)$,

$\mathrm{n}$ being positive integer, such that $n>q$.

The general class of multivariable polynomials is defined by Srivastava and Garg [1987]:

$$
S_{\mathrm{L}}^{h_{1}, \ldots, h_{r}}\left[x_{1}, \ldots, x_{r}\right]=\sum_{k_{1}, \ldots, k_{r}=0}^{h_{1} k_{1}+\ldots+h_{r} k_{r} \leq \mathrm{L}}(-\mathrm{L})_{h_{1} k_{1}+\ldots+h_{r} k_{r}} A\left(\mathrm{~L} ; k_{1}, \ldots, k_{r}\right) \frac{x_{1}^{k_{1}}}{k_{1} !} \ldots \frac{x_{r}{ }^{k_{r}}}{k_{r} !},
$$

where $h_{1}, \ldots . . h_{r}$ are positive integers and the co-efficient $A\left(\mathrm{~L} ; k_{1}, \ldots . ., \mathrm{k}_{\mathrm{r}}\right),\left(\mathrm{L} ; h_{1} \in \mathrm{N} ; i=1, \ldots ., r\right)$ are arbitrary constant, real or complex.

Evidently the case $r=1$ of the polynomials (1.11).

Would correspond the polynomials given by Shrivastava [1972]

$$
S_{\mathrm{L}}^{h}[x]=\sum_{k=0}^{[L, h]} \frac{(-\mathrm{L})_{h k}}{k !} A_{\mathrm{L}, k} x^{k}\{\mathrm{~L} \in N=(0,1,2, \ldots)\}
$$

where $h$ is arbitrary positive integers and the co-efficient $A_{\mathrm{L}, k}(\mathrm{~L}, k \geq 0)$ are arbitrary constant, real or complex.

\section{Mathematical pre-requisites:-}

To establish the main result, we need the following integral of the H-function by SaigÖ[1992]: 


$$
\begin{aligned}
\left.\int_{x}^{\infty} t^{\rho-1}(t-x)^{\sigma-1} \mathrm{I}_{p_{i}, q_{i}}^{m, r} ; z t^{\mu}(t-x)^{\nu} \mid \begin{array}{l}
\left(a_{j}, e_{j}\right)_{1, n} ;\left(a_{j i}, e_{j i}\right)_{n+1, p_{i}} \\
\left(b_{j}, f_{j}\right)_{1, m} ;\left(b_{j i}, f_{j i}\right)_{m+1, q_{i}}
\end{array}\right] d t \\
=x^{\rho+\sigma-1} \mathrm{I}_{p_{i}+2, q_{i}+1 ; r}^{m+1, n+1}\left[z x^{\mu+v} \mid \begin{array}{l}
(1-\sigma, v),\left(a_{j}, e_{j}\right)_{1, n} ;\left(a_{j i}, e_{j i}\right)_{n+1, p_{i}},(1-\rho, \mu) \\
(1-\rho-\sigma, \mu+v),\left(b_{j}, f_{j}\right)_{1, m} ;\left(b_{j i}, f_{j i}\right)_{m+1, q_{i}}
\end{array}\right],
\end{aligned}
$$

where

(i) $\rho, \sigma$ are complex numbers and $\mu, v$ are positive real numbers,

(ii) $\quad|\arg z|<\frac{1}{2} A \pi$, A defined as

$$
\begin{aligned}
& A=\sum_{j=1}^{p_{i}} e_{j i}-\sum_{j=1}^{q_{i}} f_{j i}, \\
& \min \left[\operatorname{Re}\left(\frac{1-\rho-\sigma}{\mu-v}\right), \min 1 \leq j \leq m\left[\operatorname{Re}\left(\frac{b_{j}}{f_{j}}\right)\right]\right]> \\
& \max \left[-\operatorname{Re}\left(\frac{\sigma}{v}\right), \max 1 \leq j \leq N\left[\operatorname{Re}\left(\frac{a_{j}-1}{e_{j}}\right)\right]\right] .
\end{aligned}
$$

\section{Weyl Fractional Derivatives Of The Product Of Multivariable Polynomials And I-Function.}

Theorem.

Let $m, n, p_{i}$ and $q_{i}$ be non-negative integers such that $0 \leq n \leq p_{i}, 0 \leq m \leq q_{i}$ and $\sum_{j=1}^{n} e_{j}-\sum_{j=n+1}^{p_{i}} e_{j i}+\sum_{j=1}^{m} f_{j}-\sum_{j=m+1}^{q_{i}} f_{j i}>0$ together with the set of conditions (i) - (iii) given with equation (2.1).

Then, for all value of $\mathrm{q}$,

$$
\begin{aligned}
& { }_{z} W_{\infty}^{q}\left\{x^{\rho-1}(z-x)^{\sigma-1} S_{L}^{h_{1}, \ldots, h_{r}}\left\lfloor c_{1} x^{\delta_{1}}, \ldots, c_{r} x^{\delta_{r}}\right\rfloor\right. \\
& \left.\times \mathrm{I}_{p_{i}, q_{i} ; r}^{m, n}\left[y x^{\mu}(x-z)^{v} \mid \begin{array}{l}
\left(a_{j}, e_{j}\right)_{1, n} ;\left(a_{j i}, e_{j i}\right)_{n+1, p_{i}} \\
\left(b_{j}, f_{j}\right)_{1, m} ;\left(b_{j i}, f_{j i}\right)_{m+1, q_{i}}
\end{array}\right]\right\} \\
& =\frac{(-1)^{q+\sigma-1}}{\Gamma(-q)} z^{\rho+\sigma-q-\sum_{i=1}^{r} k_{i} \delta_{i}-2} \sum_{k_{1}, \ldots, k_{r}=0}^{h_{1} k_{1}+\ldots+h_{r} k_{r} \leq L}(-L) \sum_{h_{1} k_{1}+\ldots+h_{r} k_{r}} A\left(L ; k_{1}, \ldots, k_{r}\right) \frac{c_{1}^{k_{1}}}{k_{1} !} \ldots \frac{c_{r}^{k_{r}}}{k_{r} !} \\
& \times \mathrm{I}_{p_{i+2} \cdot q_{i+1} ; r}^{m+1, n+1}\left[y z^{\mu+v} \mid \begin{array}{l}
(2-\sigma+q, v),\left(a_{j}, e_{j}\right)_{1, n} ;\left(a_{j i}, e_{j i}\right)_{n+1, p_{i}},\left(1-\rho-\sum_{i=1}^{r} k_{i} \delta_{i}, \mu\right) \\
\left(2+q-\rho-\sigma-\sum_{i=1}^{r} k_{i} \delta_{i}, \mu+v\right),\left(b_{j}, f_{j}\right)_{1, m} ;\left(b_{j i}, f_{j i}\right)_{m+1, q_{i}}
\end{array}\right] .
\end{aligned}
$$

Proof : Taking left hand side of equation (3.1)and using equation (1.11), we get

$$
\begin{aligned}
& W_{\infty}^{q}\left\{x^{\rho+\sum_{i=1}^{r} k_{i} \delta_{i}-1}(z-x)^{\sigma-1} \sum_{k_{1}, \ldots, k_{r}=0}^{h_{1} k_{1}+\ldots+h_{r} k_{r} \leq L}(-L) \sum_{h_{1} k_{1}+\ldots+h_{r} k_{r}} A\left(L ; k_{1}, \ldots, k_{r}\right) \frac{c_{1}^{k_{1}}}{k_{1} !} \ldots \frac{c_{r}^{k_{r}}}{k_{r} !}\right. \\
\times \mathrm{I}_{p_{i}, q_{i} ; r}^{m, n} & {\left.\left[y x^{\mu}(x-z)^{\nu} \mid \begin{array}{l}
\left(a_{j}, e_{j}\right)_{1, n} ;\left(a_{j i}, e_{j i}\right)_{n+1, p_{i}} \\
\left(b_{j}, f_{j}\right)_{1, m} ;\left(b_{j i}, f_{j i}\right)_{m+1, q_{i}}
\end{array}\right]\right\}, }
\end{aligned}
$$


Now using equation (1.9) and definition of I - function, easily we can find the proof of equation (3.1).

For $\mathrm{q} \geq 0$ invoking the definition (1.10) the relation (3.2) further reduces to

$$
\begin{aligned}
& =\sum_{k_{1}, \ldots, k_{r}=0}^{h_{1} k_{1}+\ldots+h_{r} k_{r} \leq L}(-L) A\left(L ; k_{1}, \ldots, k_{r}\right) \frac{c_{1}^{k_{1}}}{k_{1} !} \ldots \frac{c_{r}^{k_{r}}}{k_{r} !} \frac{(-1)^{q+r+\sigma-1}}{\Gamma(r-q)} \frac{d^{r}}{d z^{r}}\left\{z^{\rho+\sigma-q+r-\sum_{i=1}^{r} k_{i} \delta_{i}-2}\right. \\
& \left.\times \mathrm{I}_{p_{i+2} \cdot q_{i+1}, r+1, n+1}\left[\begin{array}{c}
k_{1}, \ldots, k_{r}=0 \\
y z^{\mu+\nu}
\end{array} \mid \begin{array}{c}
\left(2-\sigma \underline{1}_{1} k_{1}+\ldots+h_{h}, k^{\prime}\right. \\
(2+q),\left(a_{j}, e_{j}\right)_{1, n} ;\left(a_{j i}, e_{j i}\right)_{n+1, p_{i}},\left(1-\rho-\sigma-\sum_{i=1} k_{i} \delta_{i}, \mu\right) \\
\left.\sum_{i=1}^{r} k_{i} \delta_{i}, \mu+v\right),\left(b_{j}, f_{j}\right)_{1, m} ;\left(b_{j i}, f_{j i}\right)_{m+1, q_{i}}
\end{array}\right]\right\}
\end{aligned}
$$

In replacing of ( $\mathrm{q}-\mathrm{r})$ by $\mathrm{q}$, we may obtain again

$$
\begin{aligned}
& =\frac{(-1)^{q+\sigma-1}}{\Gamma(-q)} z^{\rho+\sigma-q-\sum_{i=1}^{r} k_{i} \delta_{i}-2} \sum_{k_{1}, \ldots, k_{r}=0}^{h_{1} k_{1}+\ldots+h_{r} k_{r} \leq L}(-L) \sum_{h_{1} k_{1}+\ldots+h_{r} k_{r}} A\left(L ; k_{1}, \ldots, k_{r}\right) \frac{c_{1}^{k_{1}}}{k_{1} !} \ldots \frac{c_{r}^{k_{r}}}{k_{r} !} \\
& \times \mathrm{I}_{p_{i+2} \cdot q_{i+1} ; r}^{m+1, n+1}\left[y z^{\mu+v} \mid \begin{array}{l}
(2-\sigma+q, v),\left(a_{j}, e_{j}\right)_{1, n} ;\left(a_{j i}, e_{j i}\right)_{n+1, p_{i}},\left(1-\rho-\sum_{i=1}^{r} k_{i} \delta_{i}, \mu\right) \\
\left(2+q-\rho-\sigma-\sum_{i=1}^{r} k_{i} \delta_{i}, \mu+v\right),\left(b_{j}, f_{j}\right)_{1, m} ;\left(b_{j i}, f_{j i}\right)_{m+1, q_{i}}
\end{array}\right] .
\end{aligned}
$$

Special Case : If we put $\mathrm{r}=1$ in the general call of multivariable polynomials given by Srivastava and Garg [1987] reduces to the polynomials given by Srivastava [1972] and I- function reduces into Fax's H - function as follows :

$$
{ }_{z} W_{\infty}^{q}\left\{{ }_{z} W_{\infty}^{q}\left\{x^{\rho-1}(z-x)^{\sigma-1} S_{\mathrm{L}}^{h}\left[x^{k}\right] \times H_{p, q}^{m, n}\left[y x^{\mu}(x-z)^{\nu} \mid \begin{array}{l}
\left(a_{1}, e_{1}\right)_{1, p} \\
\left(b_{1}, f_{1}\right)_{1, q}
\end{array}\right]\right\}\right.
$$

$=\frac{(-1)^{q+\sigma-1}}{\Gamma(-q)} z^{\rho+\sigma-q-k-2} \sum_{k=0}^{[L, k]} \frac{(-\mathrm{L})_{h k}}{k !} A_{\mathrm{L}, k} \times H_{p+2, q+1}^{m+1, n+1}\left[y z^{\mu+v} \mid \begin{array}{c}(2-\sigma+q, v),\left(a_{j}, e_{j}\right)_{1, p},(1-\rho-k, \mu) \\ (2+q-\rho-\sigma-k, \mu+v),\left(b_{j}, f_{j}\right)_{1, q}\end{array}\right]$.

Replacing $v$ by $-v$ equation (3.3) correspond to the following result according as $\mu>v, \mu<v$ and $\mu=v$, i.e. for $\mu$ $>\mathrm{v}$

$$
\begin{aligned}
& { }_{z} W_{\infty}^{q}\left\{\begin{array}{rl|l}
z^{2} W_{\infty}^{q}\left\{x^{\rho-1}(z-x)^{\sigma-1} S_{\mathrm{L}}^{h}\left[x^{k}\right] \times H\right. & m, n \\
p, q
\end{array}\left[y x^{\mu}(x-z)^{-\nu} \mid \begin{array}{l}
\left(a_{1}, e_{1}\right)_{1, p} \\
\left(b_{1}, f_{1}\right)_{1, q}
\end{array}\right]\right\} \\
& =\frac{(-1)^{q+\sigma-1}}{\Gamma(-q)} z^{\rho+\sigma-q-k-2} \sum_{k=0}^{[L, h]} \frac{(-\mathrm{L})_{h k}}{k !} A_{\mathrm{L}, k} \\
& \times H_{p+1, q+2}^{m+2, n}\left[y z^{\mu-v} \mid \begin{array}{c}
\left(a_{j}, e_{j}\right)_{1, p},(1-\rho-k, \mu) \\
(2+q-\rho-\sigma-k, \mu-v),(\sigma-q-1, v),\left(b_{j}, f_{j}\right)_{1, q}
\end{array}\right]
\end{aligned}
$$

For $\mu<v$

$$
\begin{aligned}
& { }_{z} W_{\infty}^{q}\left\{\begin{aligned}
z^{2} W_{\infty}^{q}\left\{x^{\rho-1}(z-x)^{\sigma-1} S_{\mathrm{L}}^{h}\left[x^{k}\right] \times H\right. \\
p, q
\end{aligned}\left[\begin{array}{l|l}
y x^{\mu}(x-z)^{-v} & \left(a_{1}, e_{1}\right)_{1, p} \\
\left(b_{1}, f_{1}\right)_{1, q}
\end{array}\right]\right\} \\
& =\frac{(-1)^{q+\sigma-1}}{\Gamma(-q)} z^{\rho+\sigma-q-k-2} \sum_{k=0}^{[L, h]} \frac{(-\mathrm{L})_{h k}}{k !} A_{\mathrm{L}, k} \\
& \times H_{p+2, q+1}^{m+1, n+1}\left[y z^{\mu-v} \mid \begin{array}{c}
(\rho+\sigma-q+k-1, v-\mu),\left(a_{j}, e_{j}\right)_{1, p},(1-\rho-k, \mu) \\
(\sigma-q-1, v),\left(b_{j}, f_{j}\right)_{1, q}
\end{array}\right]
\end{aligned}
$$

and $\mu=v$ 


$$
\begin{gathered}
{ }_{z} W_{\infty}^{q}\left\{z_{\infty}\left\{x^{\rho-1}(z-x)^{\sigma-1} S_{\mathrm{L}}^{h}\left[x^{k}\right] \times H^{m, n}\left[y x^{\mu}(x-z)^{-v} \mid \begin{array}{l}
\left(a_{1}, e_{1}\right)_{1, p} \\
\left(b_{1}, f_{1}\right)_{1, q}
\end{array}\right]\right\}\right. \\
=\frac{(-1)^{q+\sigma-1} \Gamma(2-q-\rho-k)}{\Gamma(-q)} z^{\rho+\sigma-q-k-2} \sum_{k=0}^{[L, h]} \frac{(-\mathrm{L})_{h k}}{k !} A_{\mathrm{L}, k} \\
\times H_{p+1, q+1}^{m+1, n}\left[y\left[\begin{array}{l}
\left(a_{j}, e_{j}\right)_{1, p},(1-\rho-k, \mu) \\
(\sigma-q-1, v),\left(b_{j}, f_{j}\right)_{1, q}
\end{array}\right] .\right.
\end{gathered}
$$

Finally writing $-\mu$ instead of $\mu$, equation (3.3) yields the following results according as $\mu>v, \mu<v$ and $\mu=v$ respectively.

For $\mu>v$

$$
z_{\infty} W_{\infty}^{q}\left\{x^{\rho-1}(z-x)^{\sigma-1} S_{\mathrm{L}}^{h}\left\lfloor x^{k}\right\rfloor \times H_{p, q}^{m, n}\left[y x^{-\mu}(x-z)^{v} \mid \begin{array}{l}
\left(a_{1}, e_{1}\right)_{1, p} \\
\left(b_{1}, f_{1}\right)_{1, q}
\end{array}\right]\right\}
$$

$=\frac{(-1)^{q+\sigma-1}}{\Gamma(-q)} z^{\rho+\sigma-q-k-2} \sum_{k=0}^{[L, h]} \frac{(-\mathrm{L})_{h k}}{k !} A_{\mathrm{L}, k}$

$\times H_{p+2, q+1}^{m, n+2}\left[y z^{-\mu+v} \mid \begin{array}{c}(q-\rho-\sigma-k+1, \mu-v),(2+q-\sigma, v),\left(a_{j}, e_{j}\right)_{1, p} \\ \left(b_{j}, f_{j}\right)_{1, q},(\rho+k, \mu)\end{array}\right]$

for $\mu<v$

$$
{ }_{z} W_{\infty}^{q}\left\{{ }_{z} W_{\infty}^{q}\left\{x^{\rho-1}(z-x)^{\sigma-1} S_{\mathrm{L}}^{h}\left[x^{k}\right] \times H_{p, q}^{m, n}\left[\begin{array}{l|l}
y x^{-\mu}(x-z)^{\nu} & \left(a_{1}, e_{1}\right)_{1, p} \\
\left(b_{1}, f_{1}\right)_{1, q}
\end{array}\right]\right\}\right.
$$

$$
\begin{aligned}
& =\frac{(-1)^{q+\sigma-1}}{\Gamma(-q)} z^{\rho+\sigma-q-k-2} \sum_{k=0}^{[L, h]} \frac{(-\mathrm{L})_{h k}}{k !} A_{\mathrm{L}, k} \\
& \times H_{p++, q+1}^{m+1, n+1}\left[y z^{-\mu+v} \mid \begin{array}{c}
(2-\sigma+q, v),\left(a_{j}, e_{j}\right)_{1, p} \\
(2+q-\rho-\sigma-k, v-\mu),\left(b_{j}, f_{j}\right)_{1, q},(\rho+k, \mu)
\end{array}\right]
\end{aligned}
$$

and for $\mu=v$

$$
\begin{aligned}
& { }_{z} W_{\infty}^{q}\left\{\begin{array}{r|r}
z_{\infty} W_{\infty}^{q}\left\{x^{\rho-1}(z-x)^{\sigma-1} S_{\mathrm{L}}^{h}\left[x^{k}\right] \times H^{m, n}\right. \\
p, q
\end{array}\left[y x^{-\mu}(x-z)^{v} \mid \begin{array}{l}
\left(a_{1}, e_{1}\right)_{1, p} \\
\left(b_{1}, f_{1}\right)_{1, q}
\end{array}\right]\right\} \\
& =\frac{\Gamma(2-\rho-k-\sigma+q)(-1)^{q+\sigma-1}}{\Gamma(-q)} z^{\rho+\sigma-q-k-2} \sum_{k=0}^{[L, h]} \frac{(-\mathrm{L})_{h k}}{k !} A_{\mathrm{L}, k} \\
& \times H_{p+2, q+1}^{m+1, n+1}\left[y z^{\mu+\nu} \mid \begin{array}{c}
(2-\sigma+q, v),\left(a_{j}, e_{j}\right)_{1, p},(1-\rho-k, \mu) \\
(2+q-\rho-\sigma-k, \mu+v),\left(b_{j}, f_{j}\right)_{1, q}
\end{array}\right] .
\end{aligned}
$$

\section{Reference:-}

1. Agrawal,P.(2011). On multiple integral relations involving eneralized Mellin-Barnes type of contour integral. Tamsui Oxford Journal of Information and Mathematical Sciences. 27(4) 449 - 462 Aletheia University.

2. Fox, C. (1968). The G and H-function as Symmetrical Fourier, Trans. Amer. Math. Soc., 98, 395-429.

3. Gupta, K.C. and Jain, U.C. (1968). On the derivative of the H-functions. Proc. Nat. Acad. Sci. India Sect., A 38, 189-192. 
4. Gupta, K.C. and Soni, R.C. (2006). On a basic integral formula involving the product of the H-function and Fox H-function. J. Raj. Acad. Sci., 4(3), 157 .

5. 5.Miller, K.S. (1975). The Weyl fractional calculus, fractional calculus and its applications. Lecture Notes in Math., 457, springer-Verlag, New York, 80-89.

6. Oldhan, K.B. and Spanier, J. (1974). The fractional calculus Academic press. New York and London.

7. Ross, B. (1974). Fractional Calculus and its applications (Proceeding of the international conference held at the university of New Haven. Springer, yerlag, Berlin Heidelberg and New York.

8. SaigÖ, M., Saxena R.K. and Ram, J. (1992). On the fractional calculus operator associated with the H-function. Ganita Sandesh 6(1), 36-47

9. Srivastava, H. M. (1972) . A contour integral involving Fox's H-function. India J. Math. 14, 1 - 6.

10. Srivastava, H. M., Gupta, K.C. and Goyal, S.P. (1982). The H -function of one and two variables with applications. South Asian Publishers, New Delhi, Madras.

11. Srivastava, H. M. and Garg, M. (1987). Some integrals involving a general class of polynomials and multivariable H-function. Rev Roumaine Phy., 32, 685 - 692.

12. Saxena, V.P. (1982). Formal solution of certain new pair of Dual integral equations involving H-function. Proc. Nat. Acad. Sci. India 52, A III, 366-375. 